\title{
Differential tissue tropism of Trypanosoma cruzi strains: an in vitro study
}

\author{
Luciana O Andrade ${ }^{1 /+}$, Lúcia MC Galvão ${ }^{2,5}$, Maria de Nazareth SL Meirelles ${ }^{3}$, \\ Egler Chiari', Sergio DJ Pena ${ }^{4}$, Andrea M Macedo ${ }^{4}$
'Departamento de Morfologia ${ }^{2}$ Departamento de Parasitologia ${ }^{4}$ Departamento de Bioquímica e Imunologia, Universidade Federal de Minas Gerais, Av. Antônio Carlos 6627, 31270-901 Belo Horizonte, MG, Brasil
${ }^{3}$ Departamento de Ultraestrutura e Biologia Celular, Fundação Oswaldo Cruz-Fiocruz, Rio de Janeiro, RJ, Brasil ${ }^{5}$ Programas de Pós-Graduação em Ciências da Saúde e Ciências Farmacêuticas, Universidade Federal do Rio Grande do Norte, Natal, RN, Brasil

\begin{abstract}
We have previously demonstrated selection favoring the JG strain of Trypanosoma cruzi in hearts of BALB/c mice that were chronically infected with an equal mixture of the monoclonal JG strain and a clone of the Colombian strain, Col1.7G2. To evaluate whether cell invasion efficiency drives this selection, we infected primary cultures of BALB/c cardiomyocytes using these same T. cruzi populations. Contrary to expectation, Coll.7G2 parasites invaded heart cell cultures in higher numbers than JG parasites; however, intracellular multiplication of JG parasites was more efficient than that of Coll.7G2 parasites. This phenomenon was only observed for cardiomyocytes and not for cultured Vero cells. Double infections (Coll.7G2 $+J G)$ showed similar results. Even though invasion might influence tissue selection, our data strongly suggest that intracellular development is important to determine parasite tissue tropism.
\end{abstract}

Key words: Trypanosoma cruzi - Chagas disease - tissue tropism - cardiomyocytes

Trypanosoma cruzi, the etiological agent of Chagas disease, is an obligate intracellular parasite that can cause debilitating chronic infection in humans. T. cruzi is transmitted to vertebrate hosts via a variety of triatomine insect vectors (Araújo et al. 2009). Infection also can occur in humans via exposure to contaminated blood during pregnancy or blood transfusion, in laboratory accidents or by ingestion of contaminated food (Moncayo \& Ortiz Yanine 2006). After entry into the vertebrate host, the parasite can infect a wide variety of cells, including macrophages, smooth and striated muscle cells, fibroblasts, Schwann cells (Leite et al. 1998). Ten to 30 years after infection with T. cruzi, some patients can develop severe cardiac and/or gastrointestinal involvement, both of which are major causes of morbidity and mortality in Chagas disease (Prata 2001). The severity and prevalence of the distinct clinical forms of the disease differ among geographical regions (Prata 2001), but the cause of such clinical and epidemiological heterogeneity is not completely understood. Many studies have searched for the molecular and cellular bases of early T. cruzi infection and the associated implications for the development of the clinical forms of the disease (Villalta et al. 2008).

Experimental murine models of T. cruzi infection have been shown to faithfully mimic various aspects of Chagas disease, including the immune mechanisms involved in controlling the parasites in humans (Andersson et al. 2003). Murine models also have been used to investigate histo-

Financial support: CNPq, FAPEMIG, WHO

+ Corresponding author: luoandrade@gmail.com

Received 3 March 2010

Accepted 16 June 2010 pathological implications (Andrade \& Magalhães 1996) of infection by different $T$. cruzi strains. By experimentally infecting mice, we have previously shown (Andrade et al. 1999, 2002) a clear and pathogenically relevant differential tissue distribution of the distinct T. cruzi clonal populations Col1.7G2 and JG (belonging to the T. cruzi I and II lineages, respectively). Analyses of tissues from chronically infected mice revealed not only different preferential tissue distribution of the two parasite populations studied but also their interaction in mixed infections. In both BALB/c and DBA-2 mice, we detected parasites in mouse hearts infected with either the Col1.7G2 clone or the JG strain in isolation. However, in mice simultaneously infected by both parasite populations, the heart tissue was parasitized exclusively by the JG strain (Andrade et al. 1999, 2002).

To improve our understanding of the mechanisms involved in this selection among $T$. cruzi populations during colonization of different host tissues, we investigated the profile of infection and development of JG and Col1.7G2 T. cruzi clonal populations in primary cultures of cardiomyocytes produced from the F1 embryos of BALB/c x DBA-2 crosses. We used F1 hybrid embryos because the progeny was larger than that of intra-strain crosses and the same parasite tissue tropism pattern emerged in both BALB/c and DBA-2 mice (Andrade et al. 1999). Primary cultures of cardiomyocytes were prepared from BALB/c x DBA-2 F1 mouse embryos after 18-19 days of gestation. Females were anesthetized and embryos were removed and processed for heart extraction according to the specifications of the Conselho Nacional de Controle de Experimentação Animal (the Brazilian institution regulating animal experimentation). Hearts were then processed for cardiomyocyte isolation as previously described (Meirelles et al. 1986). After $24 \mathrm{~h}$ of culture, cardiomyocytes were exposed to $T$. cruzi overnight $(\sim 18 \mathrm{~h})$ at a multiplicity of infec- 
A

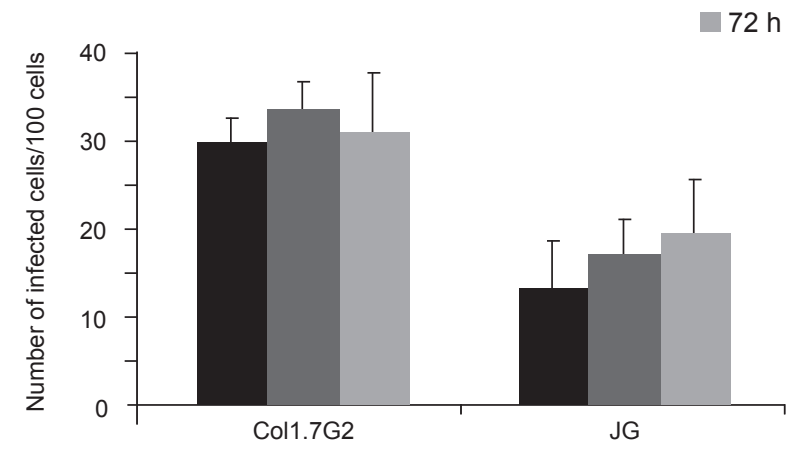

C
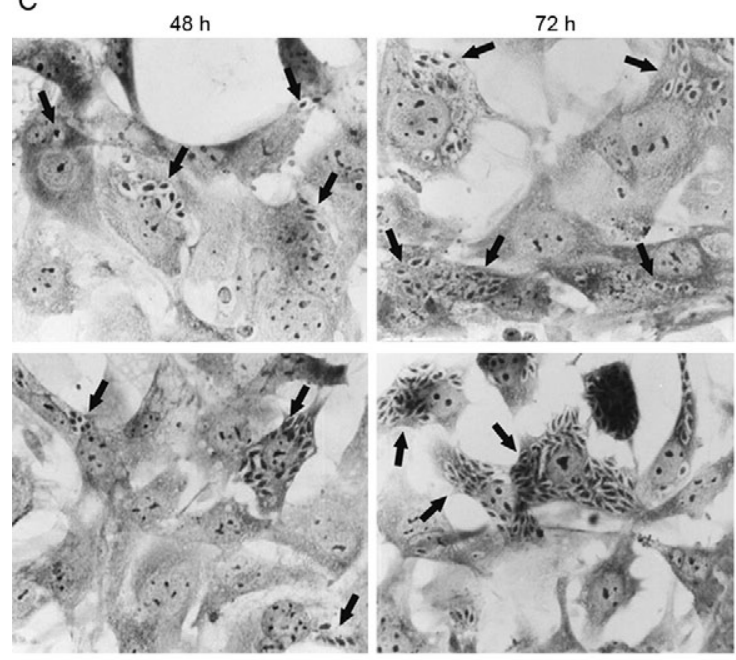

$\mathrm{CM}$
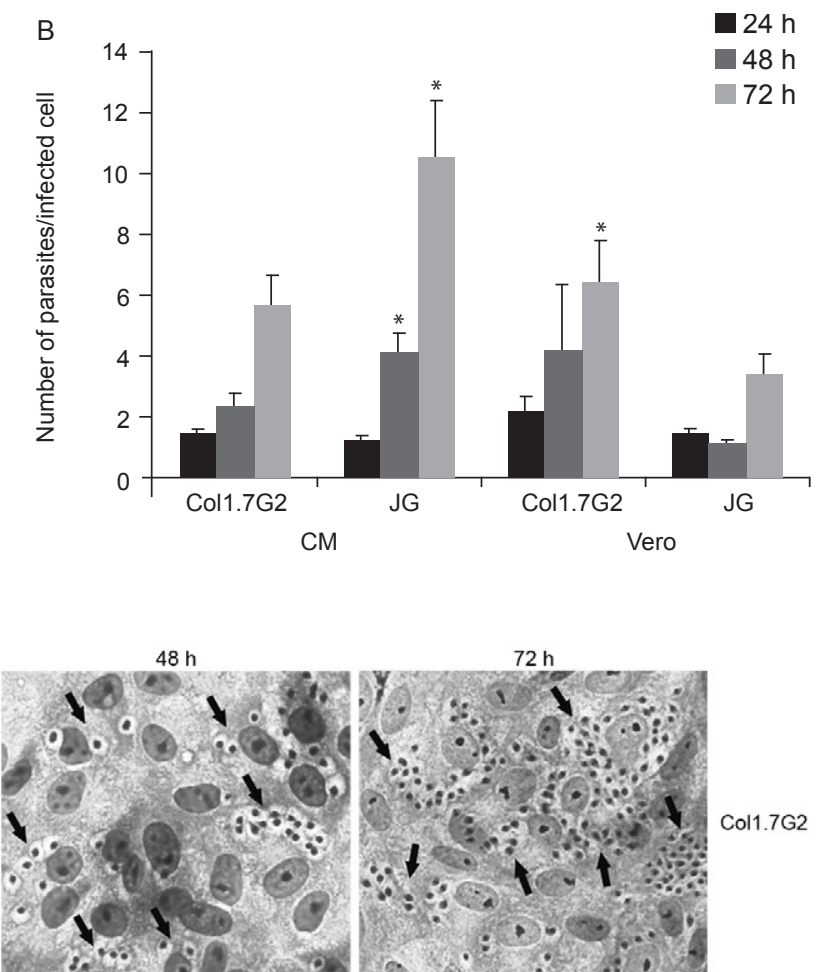

Col1.7G2
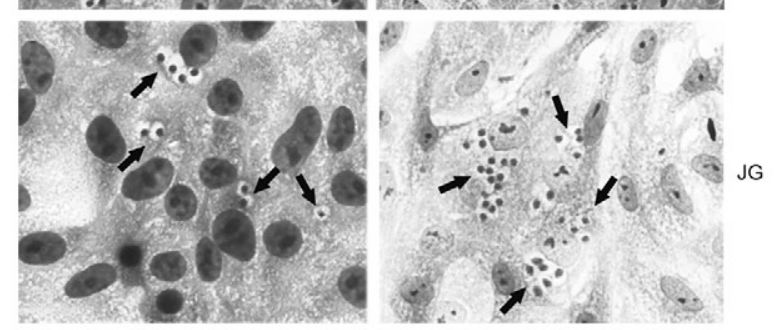

Vero

Fig. 1: cell infection assays in primary cultures of cardiomyocytes and Vero cells using two monoclonal populations of Trypanosoma cruzi, Col1.7G2 and JG. After $24 \mathrm{~h}$ of parasite exposure, cells were washed with phosphate buffered saline to eliminate extracellular parasites and either fixed with paraformaldehyde or re-incubated in media before fixation. A: graph shows the number of infected cells in 100 counted cells for cardiomyocyte cultures exposed to JG or Col1.7G2; B, C: comparison between JG and Col1.7G2 intracellular development within cardiomyocytes (CM) and Vero cells. The graph shows the number of intracellular parasites per infected cell for each time point. The pictures illustrate the data obtained for each culture at 48 and $72 \mathrm{~h}$ of infection in either cardiomyocytes or Vero cells (arrows indicate amastigote nests). The data represent the mean of triplicates \pm standard deviation and the asterisks indicate statistically significant differences $(\mathrm{p}<0.05$, Student's $t$ test) between JG and Col1.7G2 for Vero or cardiomyocytes infected cultures.

tion (MOI) of 10, washed with phosphate buffered saline (PBS) to eliminate extracellular parasites and either fixed with paraformaldehyde or re-incubated with media before fixation. Fixed cells in coverslips were stained with Giemsa and counted under a microscope for T. cruzi infection.

Behavioral differences between the two T. cruzi clonal populations were detected during in vitro infection of the primary cultures of cardiomyocytes. An infection rate of $30 \%$ was observed when cardiomyocytes were exposed to Col1.7G2 trypomastigotes; in contrast, only $15 \%$ of the cells were infected when cardiomyocyte cultures were exposed to JG trypomastigotes (Fig. 1A). Prima facie, these results seemed to differ from observations of in vivo infections of BALB/c and DBA-2 mice, in which the JG strain predominated in the hearts of chronically double-infected animals. However, analyses of primary cardiomyocyte cultures after 48 and $72 \mathrm{~h}$ of infection (corresponding to periods of parasite multiplication inside host cells) presented a different scenario. The number of intracellular parasites increased more quickly in cultures infected with JG trypomastigotes compared to cultures infected with Col1.7G2 (Fig. 1B, C). An 8-fold increase in the number of JG intracellular parasites occurred between 24-72 $\mathrm{h}$ of cardiomyocyte infection, compared to a less than 4-fold increase for cardiomyocyte cultures infected with Col1.7G2. The average number of intracellular JG parasites per infected cell was at least twice the number found in Col1.7G2-infected cultures.

In order to test the specificity of this behavior and see whether it was due to parasite interaction with cardiomyo- 
cytes rather than an in vitro effect, we performed the same infection assays in cultures of Vero cells (kidney epithelial cell line). Vero cells were exposed to $T$. cruzi overnight ( $\sim 18 \mathrm{~h})$ at a MOI of 10 , washed with PBS to eliminate extracellular parasites and processed for microscopy as described for the cardiomyocytes above. In Vero cells, the number of intracellular parasites in Col1.7G2-infected cultures was greater at all time points, indicating that the cells were invaded more effectively and were more permissive of intracellular development by the Col1.7G2 clone compared to the JG strain (Fig. 1B, C). A three-fold increase in the number of intracellular parasites between 24-72 h of infection was observed for Vero cultures exposed to Col1.7G2. Vero cells exposed to JG trypomastigotes showed not only lower numbers of infected cells, but also slower rates of intracellular parasite multiplication. The ratio of intracellular parasite numbers at 72 and $24 \mathrm{~h}$ revealed only about a two-fold increase for Vero cultures infected with the JG strain (Fig. 1B).

We also analyzed primary cardiomyocyte cultures infected simultaneously with both $T$. cruzi populations (double-infected cultures). Cardiomyocytes were exposed overnight to an equal mixture of both Col1.7G2 and JG T. cruzi populations at a MOI of 10 (5 of each $T$. cruzi population) and washed with PBS to eliminate extracellular parasites and either collected for analysis or left in media for 48 or $72 \mathrm{~h}$ before processing. In this analysis, cells were not fixed with paraformaldehyde, but instead were subjected to alkaline lysis and underwent low-stringency single specific primer polymerase chain reaction (LSSPPCR) parasite characterization as described previously (Andrade et al. 1999). Briefly, LSSP-PCR characterization required polymerase chain reaction (PCR) amplification of the 330-bp fragment of the parasite kinetoplast DNA (kDNA) minicircle. The amplification product was used in the LSSP-PCR reaction, which was performed using fluorescent primers and parasite percentages estimated in a fluorescent automated DNA sequencer (ALF, Pharmacia Biotech, Uppsala, Sweden) were plotted to show the ratio of Col1.7G2 to JG infection as a function of time.

Since we did not have a morphological marker which would enable us to distinguish between JG and Col1.7G2 intracellular parasites, we quantified parasite abundance for each T. cruzi population using the LSSP-PCR technique as described for in vivo studies (Andrade et al. 1999). The LSSP-PCR technique is based on gene signatures, wich in this case correspond to the hypervariable region of T. cru$z i$ kDNA (Pena et al. 1994, Vago et al. 1996). The LSSPPCR results indicate that similar abundances of both parasite populations were present in double-infected primary cultures after $24 \mathrm{~h}$ of parasite exposure. However, an increase in the ratio of JG to Coll.7G2 was observed after 48 and $72 \mathrm{~h}$ (Fig. 2), affirming the cell-specific selection behavior observed in cardiomyocytes.

Similar observations of differences in infectivity of myoblasts by distinct $T$. cruzi populations have been described (Araújo et al. 1986). These authors showed that in the first $24 \mathrm{~h}$ of parasite-host cell interaction, T. cruzi $\mathrm{CL}$ and Colombian strains, generally considered myotropic (Melo \& Brener 1978, Camandaroba et al. 2001), were much more infective to myoblasts than the T. cruzi $\mathrm{Y}$ strain, considered macrophagotropic (Melo \& Brener 1978).
Our data further suggest that not only parasite interaction and invasion processes but also the intracellular development of parasites are important in determining $T$. cruzi tissue colonization. What determines this rate of intracellular multiplication remains to be studied. It is likely that the kinetics of vacuole escape and amastigote differentiation influence intracellular development and these kinetics might be determined by both host and parasite genomes. Trans-sialidase activity might be responsible for early parasitophorous vacuole escape and faster parasite development (Rubin-de-Celis et al. 2006). Whether differences in parasite sialidase activity and host cell sialic acid availability are enough to account for observed differences remains to be tested. Recently, we showed that this tissue selection seems to be linked to the major histocompatibility complex (MHC) locus, probably due to genes in linkage disequilibrium with the MHC (Freitas et al. 2009). Parasite entry and/or escape from a vacuole likely trigger some specific signaling pathway dependent on host cell conditions, which determines parasite intracellular development. In fact, T. cruzi can internalize host cell TGF $\beta$ during its amastigote stage; this process can regulate parasite intracellular development and might drive the differentiation of amastigotes into trypomastigotes (Waghabi et al. 2005a, b, 2007). Thus, distinct T. cruzi strains could differ in their stimulation of production and/ or uptake of TGF $\beta$ molecules, which could influence the replication rate of the parasite. Moreover, T. cruzi infection of cardiomyocytes can stimulate production of diverse chemokines and cytokines by the cardiomyocytes, including $T N F \alpha$ and $I L-1 \beta$ (Machado et al. 2000, 2008). In the presence of IFN, these cytokines lead to the production of nitric oxide (NO), which interferes with parasite viability. Ba et al. (2010) showed that infection of cardiomyocytes also can influence their production of reactive

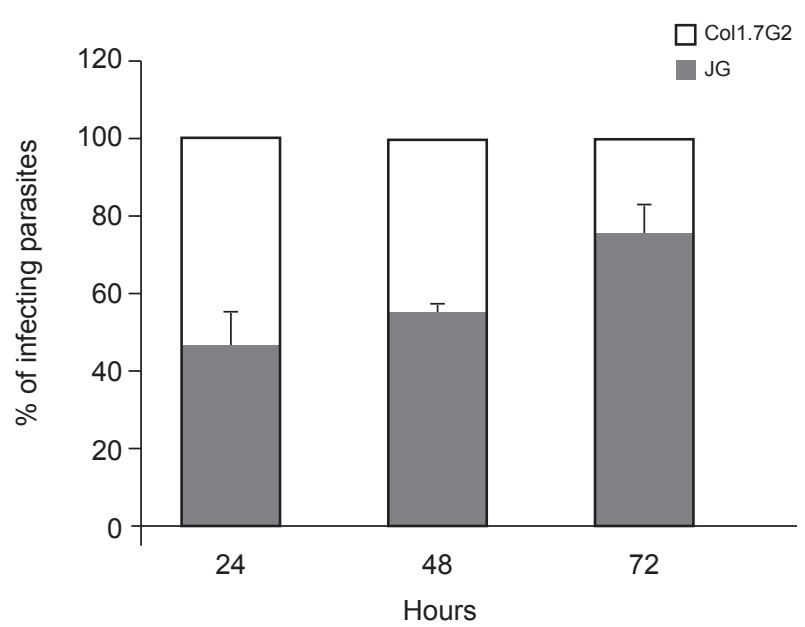

Fig. 2: evaluation of intracellular development of JG and Col1.7G2 during co-infection in cardiomyocytes exposed to an equal mixture of both Trypanosoma cruzi populations. Cultures were submitted to the same infection protocol described in Fig. 1. However, cells were subjected to alkaline lysis instead of the fixative and infecting parasites were characterized using the low-stringency single specific primer polymerase chain reaction technique. The graph shows the percentage of Col1.7G2 (white bars) in relation to JG (black bars) with time of culture. 
oxygen species (ROS), which in turn leads to enhanced expression of $T N F \alpha$ and $I L-1 \beta$. Cytokine production by cardiomyocytes, as well as production of NO and/or ROS, might then affect viability and/or intracellular multiplication of parasites. These possible selection mechanisms currently are being tested.

The results presented here provide an explanation for previously published data (Andrade et al. 1999) showing a predominance of JG in heart tissue of BALB/c and DBA-2 mice when co-infected with Col1.7G2. These results also emphasize the role of the parasite in determining tissue tropism. However, it is important to note that host immune response might modulate this tropism by varying both the number of tissue-bound as well as circulating parasites that are available for infection.

\section{REFERENCES}

Andersson J, Englund P, Sunnemark D, Dahlstedt A, Westerblad H, Nennesmo I, Orn A, Lundberg IE 2003. CBA/J mice infected with Trypanosoma cruzi: an experimental model for inflammatory myopathies. Muscle Nerve 27: 442-448.

Andrade LO, Machado CR, Chiari E, Pena SD, Macedo AM 1999. Differential tissue distribution of diverse clones of Trypanosoma cruzi in infected mice. Mol Biochem Parasitol 100: 163-172.

Andrade LO, Machado CR, Chiari E, Pena SD, Macedo AM 2002. Trypanosoma cruzi: role of host genetic background in the differential tissue distribution of parasite clonal populations. Exp Parasitol 100: 269-275.

Andrade SG, Magalhães JB 1996. Biodemes and zymodemes of Trypanosoma cruzi strains: correlations with clinical data and experimental pathology. Rev Soc Bras Med Trop 30: 27-35.

Araújo CA, Waniek PJ, Jansen AM 2009. An overview of Chagas disease and the role of triatomines on its distribution in Brazil. Vector Borne Zoonotic Dis 9: 227-234.

Araújo Jorge TC, Barbosa HS, Moreira AL, De Souza W, Meirelles MN 1986. The interaction of myotropic and macrophagotropic strains of Trypanosoma cruzi with myoblasts and fibers of skeletal muscle. Z Parasitenkd 72: 577-584.

Ba X, Gupta S, Davidson M, Garg NJ 2010. Trypanosoma cruzi induces the reactive oxygen species-PARP-1-RelA pathway for upregulation of cytokine expression in cardiomyocytes. $\mathrm{J}$ Biol Chem 285: 11596-11606.

Camandaroba EL, Campos RF, Magalhães JB, Andrade SG 2001. Clonal structure of Trypanosoma cruzi Colombian strain (biodeme Type III): biological, isoenzymic and histopathological analysis of seven isolated clones. Rev Soc Bras Med Trop 34: 151-157.

Freitas JM, Andrade LO, Pires SF, Lima R, Chiari E, Santos RR, Soares M, Machado CR, Franco GR, Pena SD, Macedo AM 2009. The MHC gene region of murine hosts influences the differential tissue tropism of infecting Trypanosoma cruzi strains. PLoS One 4: e5113.

Leite MF, Moyer MS, Andrews NW 1998. Expression of the mamma- lian calcium signaling response to Trypanosoma cruzi in Xenopus laevis oocytes. Mol Biochem Parasitol 92: 1-13.

Machado FS, Martins GA, Aliberti JC, Mestriner FL, Cunha FQ, Silva JS 2000. Trypanosoma cruzi-infected cardiomyocytes produce chemokines and cytokines that trigger potent nitric oxidedependent trypanocidal activity. Circulation 102: 3003-3008.

Machado FS, Souto JT, Rossi MA, Esper L, Tanowitz HB, Aliberti J, Silva JS 2008. Nitric oxide synthase-2 modulates chemokine production by Trypanosoma cruzi-infected cardiac myocytes. Microbes Infect 10: 1558-1566.

Meirelles MN, de Araujo-Jorge TC, Miranda CF, de Souza W, Barbosa HS 1986. Interaction of Trypanosoma cruzi with heart muscle cells: ultrastructural and cytochemical analysis of endocytic vacuole formation and effect upon myogenesis in vitro. Eur J Cell Biol 41: 198-206.

Melo RC, Brener Z 1978. Tissue tropism of different Trypanosoma cruzi strains. J Parasitol 64: 475-482.

Moncayo A, Ortiz Yanine MI 2006. An update on Chagas disease (human American trypanosomiasis). Ann Trop Med Parasitol 100: 663-677.

Pena SD, Barreto G, Vago AR, De Marco L, Reinach FC, Dias Neto E, Simpson AJ 1994. Sequence-specific "gene signatures" can be obtained by PCR with single specific primers at low stringency. Proc Natl Acad Sci USA 91: 1946-1949.

Prata A 2001. Clinical and epidemiological aspects of Chagas disease. Lancet Infect Dis 1: 92-100.

Rubin-de-Celis SS, Uemura H, Yoshida N, Schenkman S 2006. Expression of trypomastigote trans-sialidase in metacyclic forms of Trypanosoma cruzi increases parasite escape from its parasitophorous vacuole. Cell Microbiol 8: 1888-1898.

Vago AR, Macedo AM, Oliveira RP, Andrade LO, Chiari E, Galvão LM, Reis D, Pereira ME, Simpson AJ, Tostes S, Pena SD 1996. Kinetoplast DNA signatures of Trypanosoma cruzi strains obtained directly from infected tissues. Am J Pathol 149: 2153-2159.

Villalta F, Madison MN, Kleshchenko YY, Nde PN, Lima MF 2008. Molecular analysis of early host cell infection by Trypanosoma cruzi. Front Biosci 13: 3714-3734.

Waghabi MC, Keramidas M, Bailly S, Degrave W, Mendonça-Lima L, Soeiro M de N, Meirelles M de N, Paciornik S, Araújo-Jorge TC, Feige JJ 2005a. Uptake of host cell transforming growth factor-beta by Trypanosoma cruzi amastigotes in cardiomyocytes: potential role in parasite cycle completion. Am J Pathol 167: 993-1003.

Waghabi MC, Keramidas M, Calvet CM, Meuser M, de Nazaré C Soeiro M, Mendonça-Lima L, Araújo-Jorge TC, Feige JJ, Bailly S 2007. SB-431542, a transforming growth factor beta inhibitor, impairs Trypanosoma cruzi infection in cardiomyocytes and parasite cycle completion. Antimicrob Agents Chemother 51: 2905-2910.

Waghabi MC, Keramidas M, Feige JJ, Araujo-Jorge TC, Bailly S 2005b. Activation of transforming growth factor beta by Trypanosoma cruzi. Cell Microbiol 7: 511-517. 